\title{
空间 $\mathrm{X} / \gamma$ 射线探测中的关键问题
}

\section{王焕玉}

中国科学院高能物理研究所, 北京 100049

E-mail: wanghy@ihep.ac.cn

2016-11-20 收稿, 2016-12-29 修回, 2016-12-30 接受, 2017-02-28 网络版发表

\begin{abstract}
摘要 空间作为科学探索与发现的处女地、基础与工程研究的极端环境综合实验室有其独特优势. 在空间科学 探测研究中, 无论是自然物理现象研究, 还是地外资源、灾害研究和国家安全应用研究, $\mathrm{X} / \gamma$ 射线探测已经成为不 可缺少的手段. 自然界有很多未解的科学之谜, 其探索和研究与 $\mathrm{X} / \gamma$ 射线辐射探测密切相关, 基础研究关键科学问 题有: 暗物质与暗能量、超新星爆发、黑洞形成、月球与行星演化、宇宙起源等; 地外空间的可用资源; 国家安 全与空间灾害研究等等。此外，空间 X/ $\gamma$ 射线辐射探测研究与应用和地面不同，因发射与空间环境要求，空间辐射 探测仪器除了具有良好的性能外，还应具有高可靠的品质。因此，还有多个关键技术难题须攻克。空间辐射物理 研究与应用前景广阔。与美国、俄罗斯、日本等及欧洲的空间科技强国相比，我国启动较晚，投入较少，工业基础 底子薄，相对落后，尤其空间 $\mathrm{X} / \gamma$ 射线探测与应用相对基础更为薄弱，应引起高度重视，且当加大投入与支持，努 力使中国成为科学强国。
\end{abstract}

关键词空间 $\mathrm{X} / \gamma$ 射线探测, 空间科学探测, 基础科学问题, 关键技术难题

自 1895 年伦琴发现X射线以来, $\mathrm{X} / \gamma$ 射线辐射作 为有效探针和多息信使被广泛研究和应用 ${ }^{[1,2]}$. 在空 间科学探测研究中, 无论是自然物理现象基础研究, 还是地外资源、灾害研究和国家安全应用研究, X/ $\gamma$ 射线探测已经成为不可缺少的手段. 针对不同的科 学应用, 开展多目标、宽能谱段、宽视场等高精度、 高速度、高灵敏度的多模式观测, $\mathrm{X} / \gamma$ 射线技术有着不 可替代的作用.

\section{1 国内外发展态势}

空间作为科学探索与发现的处女地、基础与工程 研究的极端环境综合实验室有其独特优势, 作为不 可或缺的优质战略资源成为各国的必争之地. 俄罗 斯、美国、欧洲各国、日本、印度以及韩国、朝鲜、 伊朗、巴西等都在倾力推进自己的空间探索. 图1给 出至2040年各国空间探索的战略规划图(Breakthrough of the Year: The top 10 scientific achievements of 2014, 参见 http://www.sciencemag.org/news/2014/12; NASA 2011 Strategy Plan, 参见http://www.nasa.gov/pdf/516579 mian_NASA2011strategyPlan.pdf) ${ }^{[3 \sim 6]}$.

可以看出，从近地空间到小行星，从月球探测到 载人火星探测等一系列空间探测和利用规划，和平 利用空间将给人类发展带来巨大利益和广阔空间. 在未来的探索与发展中, 将呈现如下几个特点：一是 以探索空间科学未知为目标, 由近地向探测深空发 展; 二是挑战人类创新能力, 发展先进空间技术和探 测技术; 三是以先进智能机器人为先导发展载人航 天和地外着陆探测; 四是以近地空间轨道探测为目 标，探索和创新利用地外资源的方法与途径; 五是寻 找潜在的商业机会.

电磁辐射是认知宇宙的重要窗口。通过分析和 研究天体辐射携带的信息来研究发生在相应天体起

引用格式: 王焕玉. 空间 X/ $\gamma$ 射线探测中的关键问题. 科学通报, 2017, 62: 969-977 Wang H Y. Some key issues of X/gamma-ray detection in space (in Chinese). Chin Sci Bull, 2017, 62: 969-977, doi: 10.1360/N972016-00245 


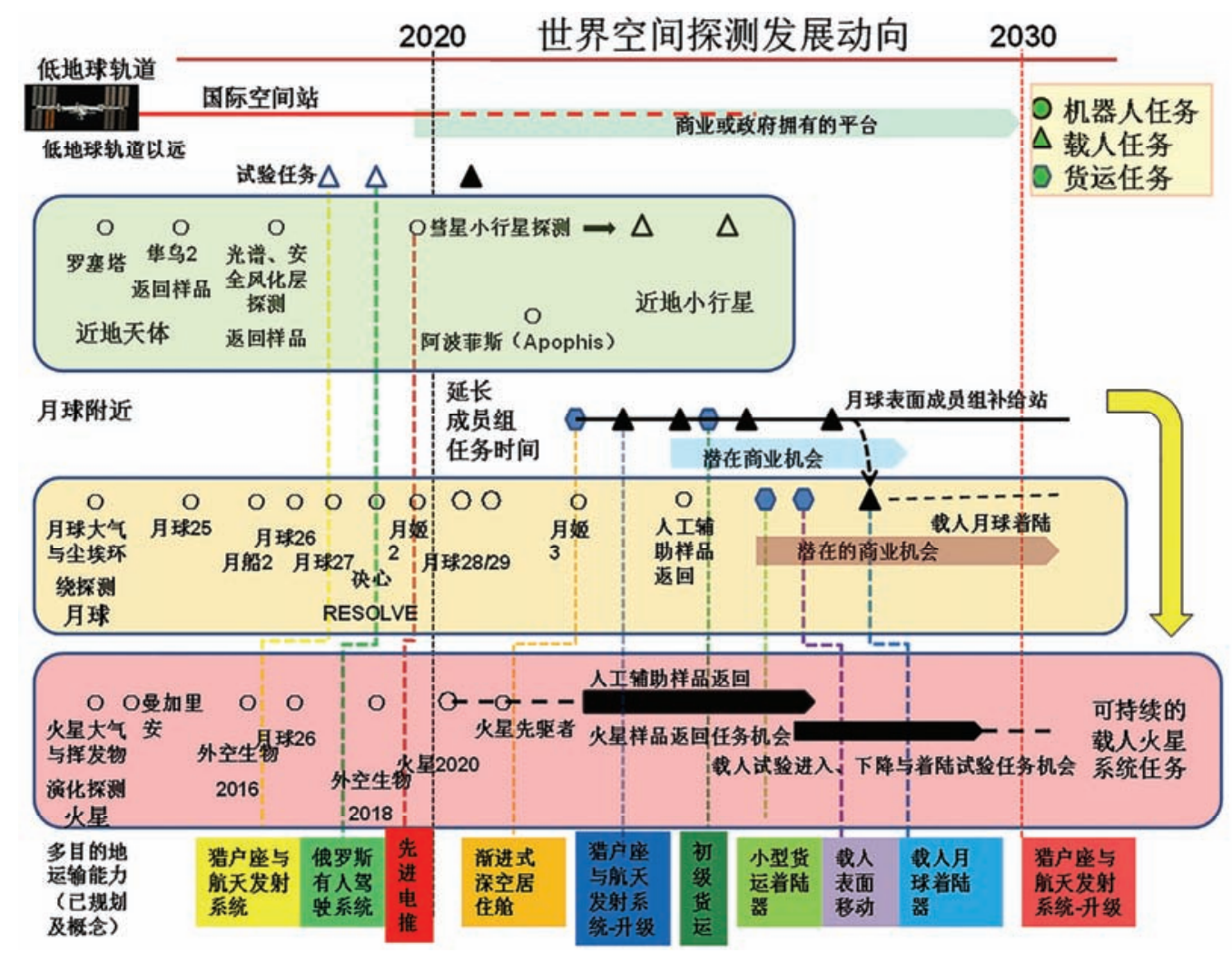

图 1 (网络版彩色)国际空间中长期探测活动发展概略

Figure 1 (Color online) International space activity outline for medium and long-term

源、演化的物理过程和规律, $\mathrm{X} / \mathrm{\gamma}$ 射线辐射观测是获取 天体信息的重要手段. 因此, 迄今为止, 人类借助发 射多颗人造卫星、高空科学气球、搭载其他航天飞行 器等观测研究空间 $X / \gamma$ 射线辐射. 如表 1 和 2 示意了国 内外典型的 $\mathrm{X} / \gamma$ 射线辐射观测实验.

从表 1 和 2 看国内外发展的动态, 自然界有很多 未解的科学之谜, 其探索和研究与 $\mathrm{X} / \mathrm{\gamma}$ 射线辐射探测 密切相关, 与此相关的关键基础物理和天体物理科 学问题有: 超新星爆发、黑洞形成、月球与行星演化、 暗物质暗能量、宇宙起源等等; 地外空间有人类所寻 受的可用资源, 以及如何鉴知、利用地外资源也是 $\mathrm{X} / \gamma$ 射线探测研究有关的热点, 例如月球及行星化学 成分、 $X / \gamma$ 射线辐射、矿物成分等等; 此外, 空间 $X / \gamma$ 射线辐射探测还可用于国家安全与空间灾害研究, 包括与国安有关的天基核爆监测、核事件监察; 与空 间灾害有关的太阳质子爆发、伽玛射线暴现象等.

和地面应用不同，空间作为 $\mathrm{X} / \gamma$ 射线辐射探测研 究的极端条件实验室有独特之处, 如空间核辐射、高 真空、微重力、引力、太阳风和星风等综合场、超宽
视场等特点, 有地面上不可替代的优势, 但同时也存 在相当大的难以预测的风险和困难，譬如受发射环 境、飞行环境和恶劣空间环境的影响, 仪器故障率 高、器件或材料寿命受限, 具有维修困难甚至不可修 复的风险, 要求空间探测仪器除了具有良好的性能 外，还应具有高可靠的品质. 因此，空间 $\mathrm{X} / \gamma$ 射线探 测还有须攻克的多项关键技术难题, 如探测仪器的 高灵敏度、超大面积、多像素; 读出电子学的低噪声、 多通道高速度、海量数据处理传输; 精密测向与距离 感知性能功能实现技术; 在轨标定、极端环境生存保 障; 系统靠性设计、软件可靠性技术问题等.

\section{2 空间 $\mathrm{X} / \gamma$ 射线辐射探测研究中的关键科 学问题}

自然界中物质相互作用过程中 $\mathrm{X} / \gamma$ 射线的产生机 制 ${ }^{[7]}$ 分为如下几类:

(1) 同步辐射(synchrotron radiation): 带电粒子 绕磁力做加速运动辐射电磁波一一磁场带电粒子轫 致辐射 $P \sim E^{2} B^{2}$; 


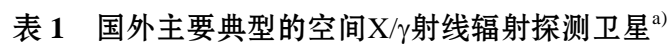

Table 1 Foreign classic space X/Gamma-ray exploration satellites

\begin{tabular}{|c|c|c|c|c|c|}
\hline 发射时间 & 组织 & 命名 & 科学目标 & 性能 & 仪器 \\
\hline $1990-01$ & 德英美 & ROSAT & NS/MC/SNR/Comet & $\begin{array}{l}0.042 \sim 2 \mathrm{keV} \\
\mathrm{d} X-120 \mu \mathrm{m} \\
\mathrm{d} \theta-5 \text { arcs }\end{array}$ & $\begin{array}{l}\text { XRT, HRI } \\
\text { PSPC, WFC }\end{array}$ \\
\hline $1995-12$ & NASA & RXTE & BH/NS/PS/XRB & $\begin{array}{l}2 \sim 250 \mathrm{keV} \\
6500 \mathrm{~cm}^{2} \\
\mathrm{~d} T: 1 \mu \mathrm{s}\end{array}$ & LAXPC NaI\&CsI \\
\hline $1995-12$ & 美欧 & SOHO & 太阳冕、太阳风、高能粒子、引力 & 紫外, 低频波 & $\begin{array}{l}\text { CDS, CELIAS, COSTEP, } \\
\text { EIT, ERNE, GOL, , } \\
\text { LASCO, MDI, SUMER, } \\
\text { SWAN, UVCS, VIRGO }\end{array}$ \\
\hline 1996-04 & 意德 & BeppoSax & GRB & $\begin{array}{l}0.1 \sim 300 \mathrm{keV}, \\
40^{\circ} \times 40^{\circ}\end{array}$ & $\begin{array}{l}\text { GSPC } \\
\text { ThinWindow } \\
\text { Nai+CsI }\end{array}$ \\
\hline 1999-07 & NASA & Chandra & 多波段多个天体目标 & $\begin{array}{l}0.04 \sim 10 \mathrm{keV}, \\
0.5 \operatorname{arcs}\end{array}$ & $\begin{array}{l}\text { Telescop } \\
\text { 3Mirror MCP+ccd }\end{array}$ \\
\hline $1999-12$ & ESA & XMMNewton & $\mathrm{DM}, \mathrm{GRB} / \mathrm{BH}$ & $\begin{array}{l}0.2 \sim 12 \mathrm{keV} \\
4425 \mathrm{~cm}^{2}\end{array}$ & Telescop: 3Mirror CCD \\
\hline $2005-07$ & 日美 & AstroE & $\mathrm{BH} / \mathrm{SNR} / \mathrm{Fe}-\mathrm{Ka}-6.4 \mathrm{kev}$ & $0.3 \sim 600 \mathrm{keV}$ & GSO, BGO SiPIN \\
\hline 2008-06 & 美欧日 & Fermi & $\mathrm{BH} / \mathrm{NS} / \mathrm{CR} / \mathrm{GRB} / \mathrm{FLR}$ & $\begin{array}{l}8 \sim 300 \mathrm{GeV} \\
6500 \mathrm{~cm}^{2} \\
\text { FO }: 1 / 15 \mathrm{sky}\end{array}$ & SiSD+W, CsI, Plastic \\
\hline $2015-10$ & 印度 & AstroSat & AGN/SNR/CGs & $\begin{array}{l}0.3 \sim 300 \mathrm{keV} \\
6000 \mathrm{~cm}^{2} \\
1^{\circ} \times 1^{\circ}\end{array}$ & CCD, LAXPC CZT \\
\hline
\end{tabular}

a) NS, 中子星; MC, 分子云; Comet, 彗星; BH, 黑洞; PS, 脉冲星; XRB, X射线暴; GRB, 伽玛射线暴; DM, 暗物质; SNR, 超新星 遗迹; CR, 宇宙线; AGN, 活动星系核; FLR, 耀斑; CGs, 星系团

表 2 我国主要空间 $X / \gamma$ 射线辐射探测简表 ${ }^{a)}$

Table 2 China main space radiation detection payloads or detectorsfor $\mathrm{X} / \gamma$ ray

\begin{tabular}{|c|c|c|c|c|c|}
\hline 发射时间 & 组织 ${ }^{b}$ & 命名 & 科学目标 & 性能 & 仪器 \\
\hline 1986- & 高能所 & Balloon-Borne-HXMT & $\mathrm{XRB} / \mathrm{GRB} / \mathrm{SNR}$ & $2 \sim 300 \mathrm{keV}$ & MWPC, $\mathrm{NaI}+\mathrm{CsI}$ \\
\hline 2001-01 & $\begin{array}{l}\text { 高能所 } \\
\text { 紫台 }\end{array}$ & $\mathrm{SZ} 2-\mathrm{X} \gamma \mathrm{D}$ & $\mathrm{XRB} / \mathrm{GRB} / \mathrm{SN}$ & $10 \sim 800 \mathrm{keV}$ & GSPC, CsI/BGO \\
\hline $2007-10$ & $\begin{array}{l}\text { 紫台 } \\
\text { 高能所 }\end{array}$ & ChangE1/X $\gamma \mathrm{D}$ & CE/Solar X/GRB & $1 \mathrm{keV} \sim 3 \mathrm{MeV}$ & $\begin{array}{l}\text { SiPIN, } \\
\text { BGO+CsI }\end{array}$ \\
\hline $2010-10$ & $\begin{array}{l}\text { 紫台 } \\
\text { 高能所 }\end{array}$ & ChangE2/X $\gamma \mathrm{D}$ & CE/Solar X/GRB & $1 \mathrm{keV} \sim 3 \mathrm{MeV}$ & $\begin{array}{l}\text { SiPIN, } \\
\mathrm{LaBr}+\mathrm{BGO}\end{array}$ \\
\hline $2013-12$ & $\begin{array}{l}\text { 高能所 } \\
\text { 紫台 }\end{array}$ & ChangE3/APXS & Lunar-CE & $1 \sim 20 \mathrm{keV}$ & $\begin{array}{l}\text { SDD } \\
\text { (原位遥控) }\end{array}$ \\
\hline $2015-12$ & $\begin{array}{l}\text { 紫台 } \\
\text { 高能所 } \\
\text { 科技大学 } \\
\text { 近物所 }\end{array}$ & $\begin{array}{l}\text { DAMPE/STK\& } \\
\text { BGO\&PSD }\end{array}$ & $\begin{array}{l}\mathrm{DM} / \mathrm{CR} \\
\mathrm{AGN} / \mathrm{SNR} / \mathrm{GRB}\end{array}$ & $\begin{array}{l}0.1 \sim 300 \mathrm{TeV} \\
95000 \mathrm{~cm}^{2} \\
1^{\circ} \times 1^{\circ}\end{array}$ & $\begin{array}{l}\mathrm{BGO} / \\
\text { Si-u-Strip/Plastic }\end{array}$ \\
\hline $2016-10$ & $\begin{array}{l}\text { 高能所 } \\
\text { 空间中心 }\end{array}$ & HXMT/LE,ME,HE & $\mathrm{BH} / \mathrm{NS} / \mathrm{PS} / \mathrm{XRB} / \mathrm{SNR}$ & $\begin{array}{l}0.1 \sim 250 \mathrm{keV}, \\
5000 \mathrm{~cm}^{2} \\
1.1^{\circ} \times 5.1^{\circ}\end{array}$ & $\begin{array}{l}\text { SCD, } \\
\text { SiPIN, } \\
\text { NaI\&CsI } \\
\text { (准直器型) }\end{array}$ \\
\hline
\end{tabular}

a) Lunar-CE, 化学元素; Solar-X, 太阳X射线; APXS, 粒子激发X谱仪; XRB, X射线暴; GRB, 伽玛射线暴; DM, 暗物质; SNR, 超 新星遗迹; $\mathrm{AGN}$, 活动星系核; b) 高能所, 中国科学院高能物理研究所; 紫台, 中国科学院紫金山天文台; 科技大学, 中国科学技术大 学; 近物所, 中国科学院近代物理研究所; 空间中心, 中国科学院国家空间科学中心组织

(2) 轫致辐射(bremsstrahlung): 带电粒子受库仑 力做加速运动辐射电磁波

$$
\Phi \sim Z^{2} Z^{2} \boldsymbol{E} / m^{2}
$$

(3) 逆康普顿散射(inverse CS): 空间高能电子将 
部分能量传递给低能光子, 使其获得能量, 而电子损 失能量;

（4）强子衰变的中性介子 $\pi^{0}\left(\pi^{0}\right.$ Decay $)$ :

$\mathrm{P}+\mathrm{P}$ 或 $\mathrm{P}+$ 核子 $\rightarrow \mathrm{P}^{\prime}+$ 核子 ${ }^{\prime}+\pi^{+}+\pi^{-}+\pi^{0}\left(\pi^{0} \rightarrow \gamma+\gamma\right)$;

(5) 物质-反物质湮灭: 同光子产生粒子对方式, 带电粒子与自己的反粒子湮灭变成光子(能量)

(6) 核衰变:

$$
\mathrm{e}^{+}+\mathrm{e}^{-} \rightarrow \gamma+\gamma ;
$$

$$
\begin{gathered}
{ }^{60} \mathrm{Co} \rightarrow{ }^{60} \mathrm{Ni}^{* *}+\mathrm{e}^{-}+v_{\mathrm{e}},{ }^{60} \mathrm{Ni}^{* *} \rightarrow{ }^{60} \mathrm{Ni}^{*}+\gamma(1.17 \mathrm{MeV}) ; \\
\searrow{ }^{60} \mathrm{Ni}+\gamma(1.33 \mathrm{MeV})
\end{gathered}
$$

(7) Neutralino湮灭: 在某种特殊境况下, 中性超 对称粒子湮灭产生

$$
\mathrm{O}+\overline{\mathrm{O}} \rightarrow \gamma+\gamma(\text { 如Axion }+\mathbf{E} \mathbf{B}) \text {. }
$$

由于 $X / \gamma$ 射线不受磁场、电场等影响, 有相对强 的穿透能力, 适宜作为空间辐射爆发事件的敏感探 针, 尤其开展宽波段辐射现象探测研究.

为认识和解决自然界末解的科学谜题, 人们以直 接的、间接的、理论求证的、实验验证的等多种方式 开展研究. 然而, 受客观现实的限制, 有些研究在地 面较为合适, 有些就不合适. 比如, 我们在低能至中 高能粒子辐射物理研究时, 可以在地面通过建造超大 型加速器、对撞机或其他大型探测装置来开展精细研 究, 不必远到空间或进入深地. 但研究多场综合于极 端条件下的高能、超能的天体物理、宇宙线物理或粒 子天体物理、行星物理、太阳物理、日地环境、空间 天气等就须要到高空或深空. 这是由于(图 $2^{[8]}$ )受地球 上空大气层、热层、磁层吸收与阻挡, 除了超高能宇 宙线外, 空间中低能粒子流、 $\mathrm{X} / \gamma$ 射线很难穿越进人地
球表面，亦即使得基于 $\mathrm{X} / \gamma$ 射线辐射探测的科学研究 极为困难. 然而, 宇宙空间是大千世界万象之源, 是 不可替代的最为综合性、极端条件下的实验室, 因此 到空间开展辐射相关研究已成为人类的渴望和必然.

以恒星天体演化过程研究为例, 图 3 和 4 分别描 述了天体起源、演化、形成和凋亡的过程和 $\mathrm{X}$ 射线脉 冲星(中子星)类天体的热盘与喷流得情景. 由图3可 知, 星体(系)的演化 ${ }^{[9]}$ 是在某种条件下, 或因空间的 原初细微气花尘芮, 或直接由星风的遗流在极端条 件下形成星体或星系的源介质, 这些介质可能是气 态或尘埃的, 经由长时间特殊环境下栖徙、凝聚、演 化形成形态各异的雉星, 即少年时代的星体. 雉形的 星体在空间的极端环境中继续发展, 再历经数百上 千万年漫长的演化, 主要是原子核反应与综合过程, 星体进人青壮年时期. 接下来的数万亿年间凝聚、吸 积、并噬、活耀, 使之质量愈来愈大, 密度越来越高. 质量大到或密度达到一定程度, 或者到达某一临界 点, 星体进人调零徐老时代. 在极端环境中的老年星 体有两类结局，一类是演变成中子星、白矮星和黑洞 等致密天体. 另一类是爆炸甚至膨胀成星风, 它们或 者被加速成为我们熟知的宇宙线, 或者又富集成为 新的星球(系)介质, 进入新一轮新星演化进程, 或者 为遗迹进人空间放任“游荡”.上述演化进程的每个 环节均伴随着 $X / \gamma$ 射线产生, 对其演化过程的探索和 研究均与 $X / \gamma$ 射线辐射探测密切相关, 其中包含着新 奇的物理化学的演变机理和大尺度结构机制演化研 究. 图 4 即是用 $\mathrm{X}$ 射线谱作为探针对毫秒脉冲星 (中 子星)的演化过程的研究, 这也是粒子天体物理中采

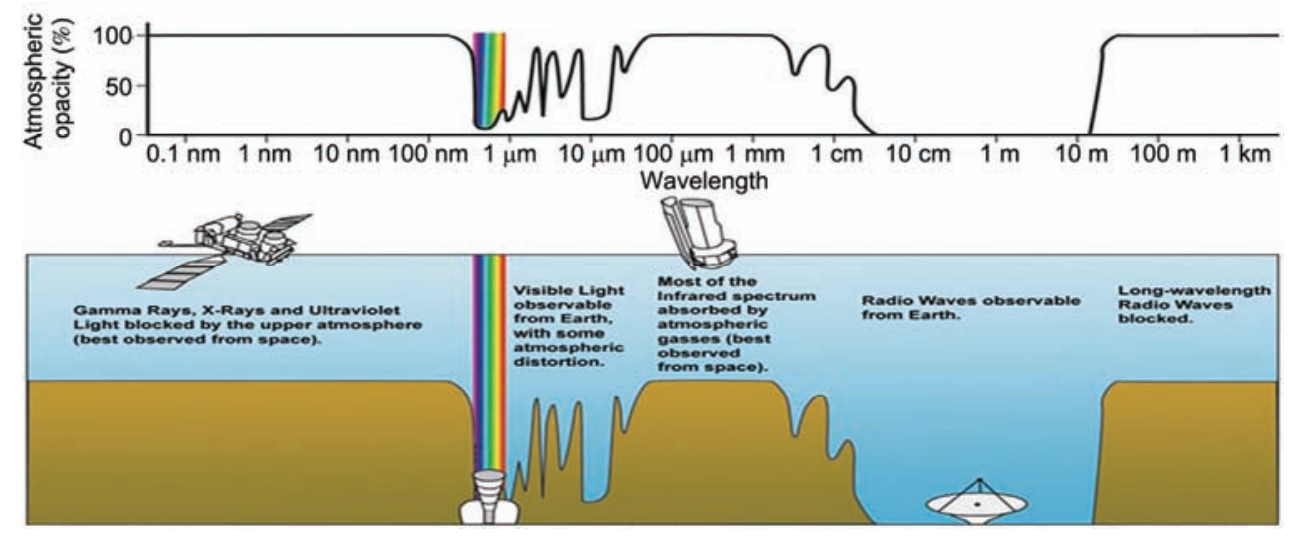

图 2 (网络版彩色)高空大气层的遮挡效应与探测窗口 ${ }^{[8]}$.

Figure 2 (Color online) The atmosphere opacity as a function of the wavelength is presented (upper part). Opacity is represented by the percentage of electromagnetic radiation, which does not reach the Ground ${ }^{[8]}$ 


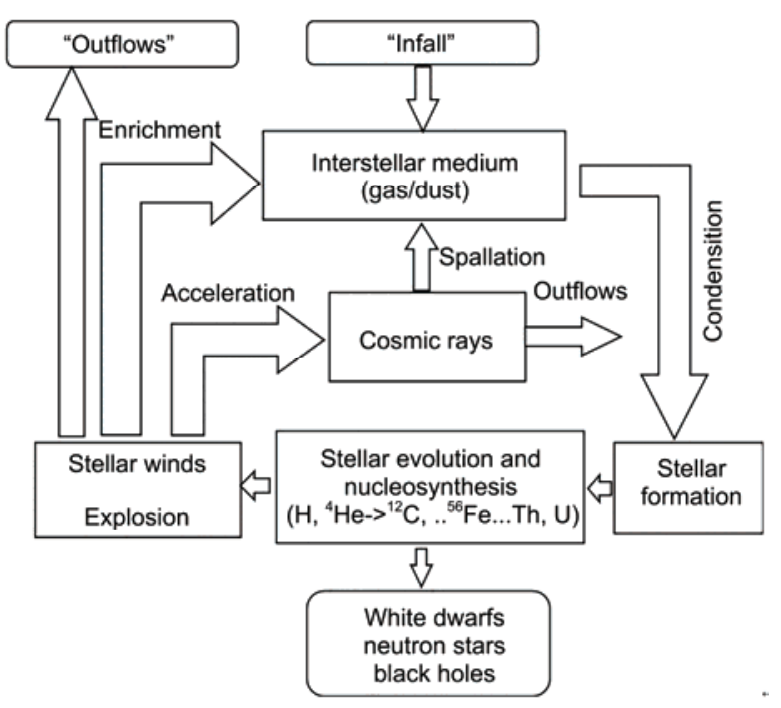

图 3 星系的形成与演化过程 ${ }^{[9]}$

Figure 3 Flowing chart of galaxy formation and evolution ${ }^{[9]}$

用探测 $X$ 射线方法研究宇宙中大尺度的天体的典型 之例. Falanga等科学家 ${ }^{[10,11]}$ 通过探测宽波段 $\mathrm{X}$ 射线的 能谱结构理解中子星的吸积盘表面热辐射物质和喷流 中的等离子体的变化, 解构主-伴星的系统耦合规律, 也是综合INTEGRAL和XMM等多个卫星上的X射线 观测装置的科学数据的结果.

随着航天技术进步，如卫星通讯、卫星气象预 报、载人航天等, 人类进人了太空时代, 改变着人类 的生活方式, 拓展了活动的空间. 随机发生的高能宇 宙线、空间辐射带和太阳耀斑事件是造成空间灾害重 要因素, 给航天器、航天员安全和无线通讯等造成严 重威胁. 它们中包含带电的质子、离子、电子等X, $\gamma$
等射线. 图5示意了 2000 年7月 12 15日太阳高能粒子 事件监测谱, 其中给出 $\mathrm{X}$ 射线耀斑的光变曲线. 这些 频繁的高能或高通量的X射线耀斑，通过光电效应、 康普顿散射和电子对产生的作用机制 ${ }^{[7]}$, 以及他们产 生的次级电离效应, 使空间仪器的电子学电路的元 件、部件的相关材料或与航天器的生物物质材料的内 部较长时间处于电离、高温、腐蚀等状态, 致使其性 能减退、功能失效、系统毁坏 ${ }^{[7,12 ~ 14]}$; 同时, 有可能 造成地面无线电通讯系统中断或受到严重干扰. 这 现象随机发生复杂的机理、效应和有效防护技术措施 亦成为辐射物理研究的重要内容之一.

多年的空间探测的实验以及辐射物理研究表明, 虽然人类在空间物理、空间天文、空间材料以及空间 生命活动等领域取得了一些成果，但与人类对空间 自然认知的深度和广度的需要相比只是冰山一角, 仅涉及 X, $\gamma$ 等射线观测研究的关键科学问题包括:

(1) 宇宙的元素起源和演化;

(2) 宇宙的能量和辐射起源;

（3）极端条件物理. 包括超新星爆发、黑洞形成、 月球与行星演化、暗物质暗能量、宇宙起源等等; 地 外空间有人类所寻受的可用资源等.

如何鉴知、利用的应用研究也是 $X / \gamma$ 射线探测研 究有关的热点:

（1）月球及行星化学成分、矿物成分;

(2) 空间材料科学中 $X / \gamma$ 射线辐射特征研究;

(3) 用于国家安全与空间灾害的空间 $X / \gamma$ 射线辐射 研究, 包括与国安有关的天基核爆监测、核事件监察; 与空间灾害有关的太阳质子爆发、伽玛射线暴现象等.

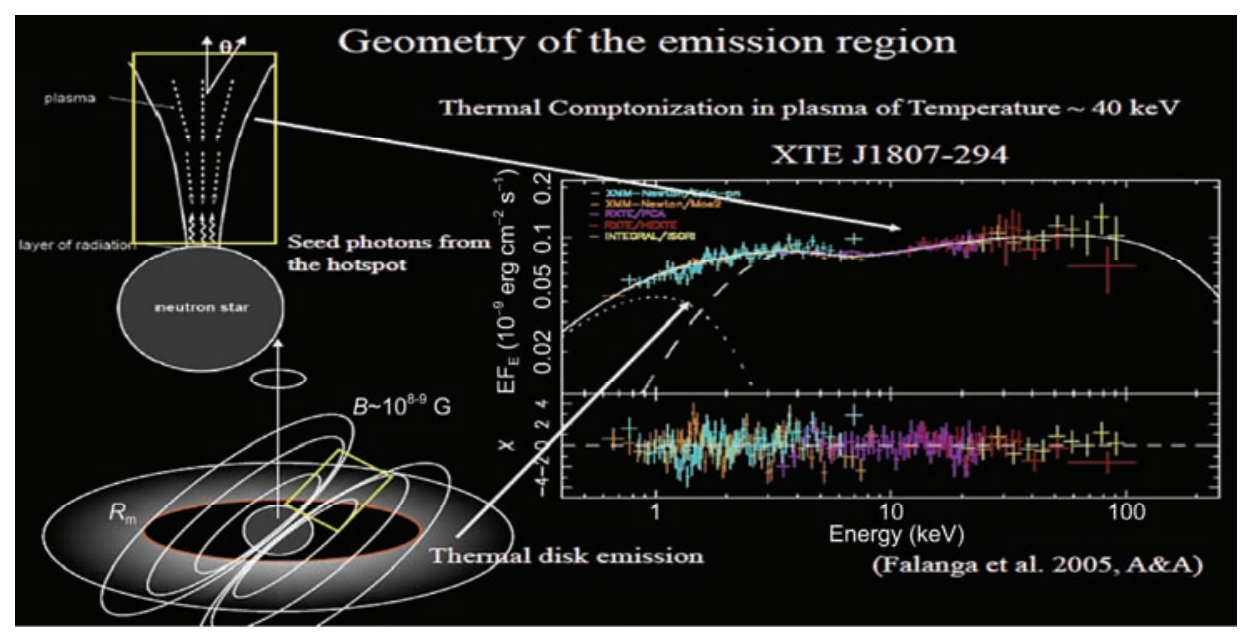

图 4 (网络版彩色) 毫秒脉冲星的吸积与喷流X射线谱 ${ }^{[10]}$

Figure 4 (Color online) XTE J1807-294 millisecond pulsar Jet and accretion of X-ray spectra ${ }^{[10]}$ 


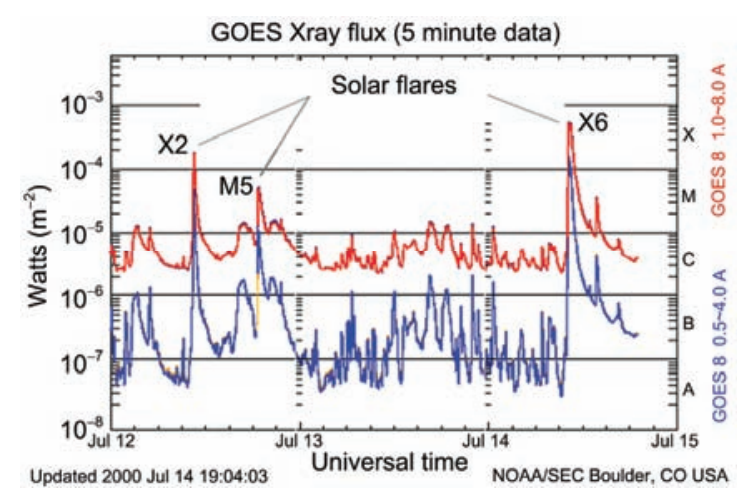

图 5 (网络版彩色) GEOS卫星 2000 年 7 月 12 至 15 日观测得到的太 阳耀斑 X 射线谱 (http://www.swpc.noaa.gov/noaa-scales-explanation; National Space Weather Strategy, Oct. 2015, http://www.whitehouse. gov/site/defaul/spaceweatherstrategy_20151028.pdf)

Figure 5 (Color online) X-ray spectra of solar flares during 12/7/200015/7/2000 observed by GOES satellite (http://www.swpc.noaa.gov/ noaa-scales-explanation; National Space Weather Strategy, Oct. 2015, http://www.whitehouse.gov/site/defaul/spaceweatherstrategy_20151028. pdf)

\section{3 空间 $\mathrm{X} / \gamma$ 射线辐射探测研究中的关键技 术问题}

如前所述，空间作为 $X / \gamma$ 射线辐射探测研究的极 端条件实验室有独特之处, 如空间辐射、高真空、微 重力等综合场、超宽视场等特点, 但是就空间探测仪 器或装置操作而言也存在相当大的风险和困难, 譬 如一旦航天器发射, 探测仪器就面临不可维修或修 复极其困难的风险. 所以, 在日益频繁的辐射物理研 究, 除了要求空间辐射探测仪器具有良好的性能外, 还应具有耐受发射环境、飞行环境和恶劣空间环境,
以及故障率低、寿命长、高可靠的品质. 因此，空间 $\mathrm{X} / \gamma$ 射线探测研究还有须攻克的多项关键技术难关. 图6给出了一个典型的空间 $\mathrm{X} / \gamma$ 射线辐射探测实验装 置的示意图 ${ }^{[8]}$. 探测装置中包括了反符合探测器、电 子-正电子转换器、径迹探测器、飞行时间探测器和 电磁量能器。一个空间探测实验一般探测的物理参 量包括，粒子种类、能量、流量、方位、角度、时间、 位置等等. 通常，空间辐射探测仪器具有高灵敏度、 大面积、多像素; 低噪声、高速度的读出电子学、海 量数据处理传输; 精密测向与距离感知、在轨标定, 极端环境生存功能; 可靠软件实施等, 通道数也往往 从几路至几十万路. 仅以国内外几个大型空间探测 装置为例, 我国的暗物质卫星上的硅阵列探测器就 有73400多路探测信号, AMS02阿尔法谱仪有30多万 路信号 ${ }^{[15]}$, 而费米卫星上的FERMI-LAT的探测器信 号多达 88 万路以上 ${ }^{[14,16]}$. 不同的科学目标对实验要 求不同, 需求的关键技术不同, 有的需要高能量分 辨, 有的要求高时间分辨, 还有的需要宽能段快速测 量等等. 面向当今高分辨大视场成像性能的空间辐射 探测载荷需求, 需要解决的关键技术主要有:

(1) 高性能的探测器(高灵敏、像素型或微条型);

(2) 低噪声、低功耗、多通道、轻小、多功能的 专用集成电路;

(3) 高质级的元器件, 包括可编程器件;

(4) 测试与对位、特殊核部件材料加工技术;

(5) 标定设备装置等.

值得特别指出的是, 在先进探测器的后端, 需要 配置高密度高性能的读出电子学和数据采集系统.

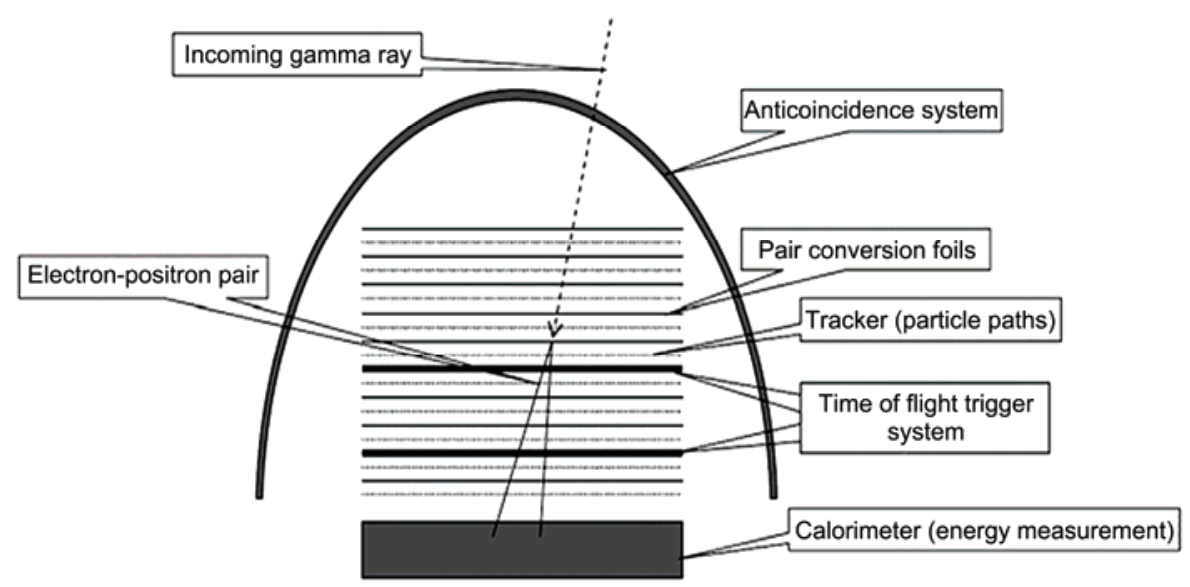

图 6 空间粒子探测谱仪示意图 ${ }^{[8,14]}$

Figure 6 The typical schematic of space particle detection spectrometer ${ }^{[8,14]}$ 
它作为探测系统的核心部件, 主要由前端信号读出、 模拟数字变换和后端的逻辑组织与判选三部分组成, 单独单片设计, 成为一个高密度高性能的小型电子 学系统 ${ }^{[14,15] 1)}$, 具有如下功能:

(1) 多路并行处理, 16或64路以上, 且每路有前 端读出、放大、成形和甄别、 $\mathrm{ADC}$ 等;

(2) 极性可调, 适应电子或孔穴读出, 或正负偏 置电压, 极性可选功能;

(3) 探测器适配, 适应不同等效(电容带来的)阻 抗探测器;

(4) 增益多档可调, 适应不同动态范围探测器系 统设计的增益分档可选;

(5) 成形时间可变, 适应不同能谱分辨率或事例 的探测系统设计的成形时间常数可选;

(6) 触发模式可选, 配置不同事例判选、获取模 式探测系统设计的分档可选;

(7) 菊花链式扩展, 适应组成更多通道、较大规 模的探测系统(但单链不大于512通道);

(8) 各道统一校正, 具有各通道统一校准(测试) 功能, 以便测试各道性能蜕变与否;

(9) 微小单路功耗. 单个通道功耗在微瓦-几十 微瓦量级, 使得供电和热设计成本降低.

采用具有这样的高性能、多功能电子学集成电路
或专用模块(ASIC)(包括前端模拟读出、后端逻辑-数 字等集成电路)与先进的可编程逻辑阵列 (firmware) 联合进行系统设计, 才可能实现体积小, 重量轻, 低 功耗, 系统紧凑, 高可靠性, 低成本的空间辐射探测 装置, 才能提高空间辐射探测装置的效率和水平, 才 能充分利用宝贵的平台资源达到科学实验目的. 图7 给出了一个单片多功能专用集成电路(ASIC)功能框 图(Gamma-IDEAS VATA系列).

值得关注的是, 我国与国际发达国家相比, 在先 进的空间辐射(粒子)探测器研制方面总体还较落后, 主要由于材料、工艺和制造技术等基础工业跟不上, 大部分材料质量、单元性能、生产规模等还有较大差 距. 硅基半导体、CZT像素(漂移室、微条)探测器; 高 纯锗、溴化镧晶体探测器等还需依靠进口. 在核心芯 片方面, 专用集成电路ASIC、几十万门及以上的大规 模可编程门阵列、微小型片上数字处理系统等尽管国 外在抗静电、抗辐照等性能方面也还不是十分理想; 但在我国此类技术尚属空白, 或为刚刚起步, 需要加 大投人和抓紧攻关, 为空间辐射物理研究的打牢技 术基础.

\section{4 结语与建议}

空间辐射物理研究与应用前景广阔, 横跨空间

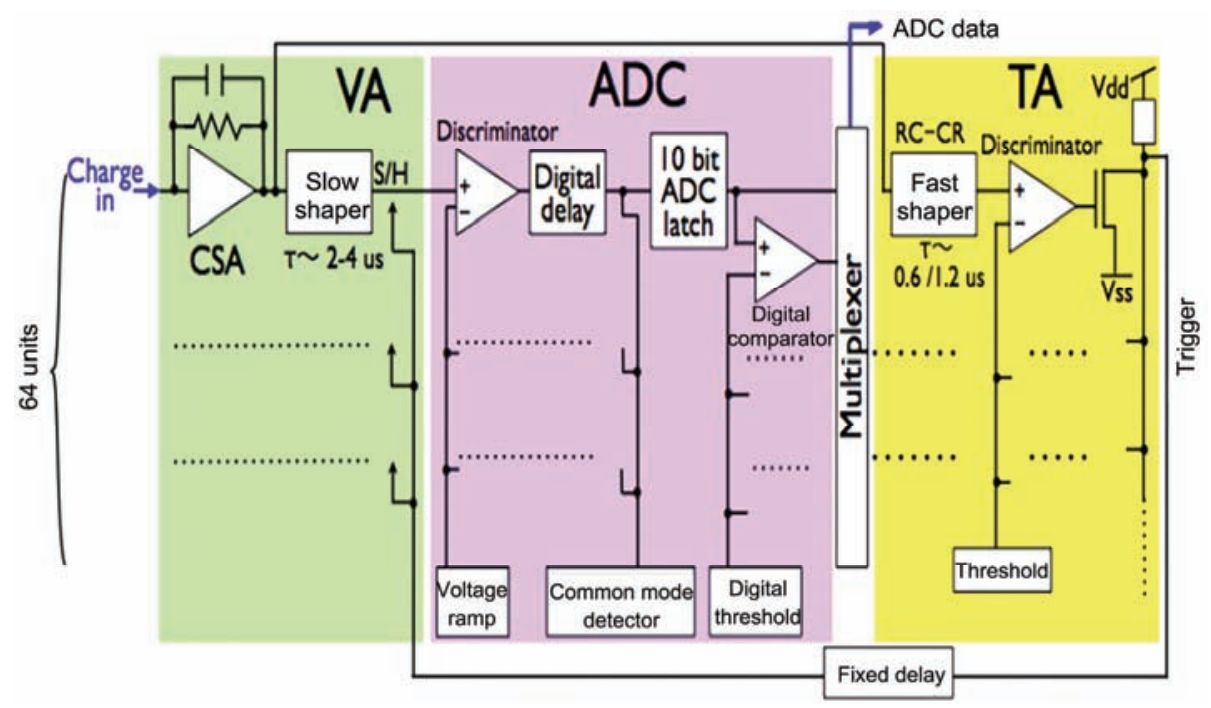

图 7 (网络版彩色)多功能专用读出集成电路框图 ${ }^{1)}$

Figure 7 (Color online) The block diagram of multifunctional application specificintegrated circuit for detector read out ${ }^{1)}$

1) Integrated Detector Electronics AS, Readout and Controller Integrated Circuits for Large Area Imaging Arrays. Http://www.ideas. no/2015/12/ideas-roic-products.pdf 
和原子核两大关键科学领域, 是涉及当前基础科学 问题和热点问题的交叉基础性学科, $\mathrm{X} / \gamma$ 射线探测是 辐射物理研究的核心内容之一. 辐射物理中 $X / \gamma$ 射线 探测研究中的基础科学问题和关键技术问题两个方 面关系密切, 需要同步发展, 协调发展, 基础科学问 题创新汼引适应复杂空间环境的关键技术进步, 先 进的技术 (包括关键工艺和系统集成技术), 支撑并推 动着关键科学问题研究与创新. 国际上空间科学研
究美国、俄罗斯、日本等及欧洲的空间科技强国起步 早、投人大、基础较好. 而我国，尽管近些年进步较 快, 可终究因起步较晚, 投人较少, 工业基础底子 薄, 仍然相对落后, 尤其空间 $X / \gamma$ 射线探测与应用相 对基础更为薄弱; 科学载荷的小型化、轻量化、智能 化不够; 大部分核心元器件、原材料依赖进口，发展 受到限制, 呼吁有关部门统筹协调, 加大投人持续支 持, 努力使我国成为科学强国.

\section{参考文献}

1 Grupen C, Cowan G, Eidelman S. Astroparticle Physics. Berlin: Springer Press, 2005

2 Phillips K J H, Feldman U, Land E, et al. Ultraviolet AND X-ray Spectroscopy of the Solar Atomosphere. Cambridge: Cambridge University Press, 2008

3 Space Mission Strategy Research Group of Chinese Academy of Sciences. 2050 Space Technology Development Roadmap (in Chinese). Beijing: Science Press, 2009 [中国科学院空间领域战略研究组. 太空之路——2050 年空间科技领域发展路线图. 北京: 科学出版社, 2009]

4 Wu J, Zhao H, Qin G, et al. Cosmic Vision 2015-2025 Space Science for The European Space Agency (in Chinese). Beijing: Space Mission Strategy Research Group of Chinese Academy of Sciences, 2007 [吴季，赵华，秦刚，等. 宇宙憧憬 2015 2025 欧洲空间局空间科 学发展计划. 北京: 中国科学院空间科学项目中长期规划研究课题组, 2007]

5 Li L, Wu J, Wang C, et al. 2006 NASA Strategy Plan (in Chinese). Beijing: Space Mission Strategy Research Group of Chinese Academy of Sciences, 2007 [李磊, 吴季, 王赤, 等. 2006 美国航空航天局战略计划. 北京: 中国科学院空间科学项目中长期规划研究课题组, 2007]

6 OuYang Z Y. Introduction to Lunar Science (in Chinese). Beijing: China Aerospace Press, 2005 [欧阳自远. 月球科学概论. 北京: 中国 宇航出版社, 2005]

7 Habing D H, Wirth J L. Anomolous photocurrent generation in transistor structures. IEEE Trans Nucl Sci, 1966, 13: 86-94

8 Spurio M. Particles and Astrophysics. Switzerland: Springer Press, 2015

9 Wong S M S. Nuclear Astrophysics. Berlin: Wiley-VCH Verlag GambH, 2007. 355-388

10 Falanga M, Bonnet J M, Ppoutanen J, et al. INTEGRAL spectroscopy of the accreting millisecond pulsar XTE J1807-294 in outburst. Astron Astrophys, 2005, 436: 647-652

11 Abdo A A, Ackermann M, Ajello M, et al. Fermi large area telescope measurements of the dffuse gamma-ray emission at intermediate latitudes. Phys Rev Lett, 2009, 103: 251101

12 Fleedwood D M. Border traps in MOS devices. IEEE Trans Nucl Sci, 1992, 39: 269-271

13 Vance E F. Shielding effectiveness of braided wire shields. IEEE Trans Elect Compat, 1975, 17: 71-77

14 Tavani M, Barbiellini G, Argon A, et al. The AGILE space mission. Nucl Instr Meth Phys Res A, 2008, 588: 52-62

15 Capell M. AMS-02 Electronics. In: Proceedings of 28th International Cosmic Ray Conference. Tokyo, 2003

16 Atwood W, Abdo A A, Ackermann M, et al. The Large Area Telescope on the Fermi mission. Astrophys J, 2009, 697: 1071-1102 
Summary for “空间 $\mathbf{X} / \gamma$ 射线探测中的关键问题”

\title{
Some key issues of X/gamma-ray detection in space
}

\author{
WANG HuanYu \\ Institute of High Energy Physics of Chinese Academy of Sciences, Beijing 100049, China \\ E-mail: wanghy@ihep.ac.cn
}

Space as a frontier of scientific exploration and discovery, basic and extreme environment engineering research laboratory has its own unique advantages. In the study of space science exploration, whether it is a natural physical phenomenon research, or outside resources, disaster and security application, X/gamma-ray detection has become an indispensable necessity.

There are many unsolved mysteries of science of nature, its exploration and research are closely related to X/gamma-ray radiation detection, key scientific issues are: supernovae, black hole formation, Lunar and planetary evolution and the origin of the universe, dark matter and dark energy; and outer space resources available; national security and space research, and so on. In addition, the space X/gamma-ray radiation detection research and application is different from the ground-based observation, as launches and space environment requirements, space radiation detection instruments not only have good performance, but also should have a high and reliable quality. Therefore, there are some of key technical problems to be overcome.

This paper consists of three main parts. In the first part, described as an integrated lab space has in the radiation field, high vacuum, microgravity, comprehensive and wide field of view, and other features, the peaceful use of space will bring enormous benefits for human development and wide space, and given in table development status in worldwide, it describes several big space nations to solve the scientific fundamental problems and key technical challenges, made a series of research strategy or projects, and gradually promote the implementation. Highlight five trends that are targeted to explore unknown space science and to develop deep space: the first is exploring the unknown space science, developing deep space exploration; the second is challenging human innovation, which is the development of advanced space technology and detection technology; the third is based on advanced intelligent robots for leading development of manned space; the fourth is to target the near-Earth orbit exploration, made innovation in methods and ways to use outside resources; the fifth point is looking for potential business opportunities.

In the second section, highlights the fundamental scientific problems in the space X/gamma-ray detection, including cosmic gamma-ray bursts, black holes, neutron stars and Supernova remnants, such as dark matter and dark energy. Meanwhile, also pointed to the space environment, planetary evolution, space electromagnetic hazards, multidisciplinary issues such as ex-territorial resources available.

In order to achieve some of the fundamental science goals designed, key technologies and processes in space experiments need to be addressed. In the third part, the space X/gamma ray radiation detection key technical challenges are described. Different science targets relay on different experiment requirements, needs of key technology are different. General speaking, physical parameters of a space radiation detection experiment include charge particles discrimination, energy detection, and flux, azimuth, angle, time, position detection as well. Usually, space radiation detection instrument has high sensitivity, and large effective area, and more pixel; low noise, and high speed of read out electronics, and mass data processing transmission; precision pointing and distance sensing and perception, and in-orbit calibration, extreme environment survival function; reliable software implementation, channel number also often from several ten of channels to around million. Technical problems to be solved are following: (1) high performance detectors (high sensitivity, pixels or micro-strip type); (2) low-noise, low-powerdissipation, multichannel, lightweight, multi-purpose ASICs; (3) high quality components, including devices; (4) test and alignment, special nuclear materials processing technologies; (5) calibration equipments and instruments.

Future prospects of space radiation physics research and application are very broad and promising. In comparison with the United States, Russia, Europe, Japan and other space technology power nations than started late in China, less investment, weak industrial base, is relatively backward, especially, X/gamma-ray detection and application of relatively weak basis, should pay more attention to, and increase investment and support efforts to make China a scientific power in space exploration.

\section{X/gamma-ray detection of space, space exploration, key science issues, key technical challenges}

doi: $10.1360 /$ N972016-00245 